\title{
Paediatric Infected Non-union of an Open Femoral Diaphyseal Fracture: The Role of Temporary Locking Plate and Bone Graft Substitute
}

\author{
Ren Yi Kow ${ }^{1,2}$, Zamri Ab Rahman ${ }^{3}$, Chooi Leng Low ${ }^{1}$, Ed Simor Khan Mor Japar Khan², Ruben Jaya Kumar ${ }^{3}$ \\ ${ }^{1}$ Hospital Tengku Ampuan Afzan, Malaysia \\ ${ }^{2}$ International Islamic University Malaysia, Malaysia \\ ${ }^{3}$ Hospital Kuala Lipis, Malaysia
}

*Corresponding author: Ren Yi Kow, International Islamic University Malaysia, Bandar Indera Mahkota, 25200 Kuantan, Pahang, Malaysia

Submission: 眥 July 09, 2018; Published: 㭗July 13, 2018

\begin{abstract}
Chronic osteomyelitis is a complication of open fractures in children especially in rural areas. It is difficult to treat, and it may lead to infected non-union. We present a case of an open fracture Gustillo-Anderson grade IIIa complicated with chronic osteomyelitis. He presented with non-union six months post-trauma after the initial surgical debridement and bone resection. He was subsequently treated successfully with a temporary $3.5 \mathrm{~mm}$ locking plate and synthetic bone graft. A temporary locking plate can stabilize the fracture site and provide additional advantage in terms of wound management and earlier joint motion in comparison with external fixation and cast.
\end{abstract}

Keywords: Infected non-union; Osteomyelitis; Locking plate; Bone graft substitute

\section{Introduction}

Chronic osteomyelitis is a complication of open fractures in children especially in rural areas. It is difficult to treat, and it may lead to infected non-union. We present a case of an open fracture

Gustillo-Anderson grade IIIa complicated with infected non-union that is successfully treated with a temporary $3.5 \mathrm{~mm}$ locking plate (LP) and synthetic bone graft.

\section{Case Report}

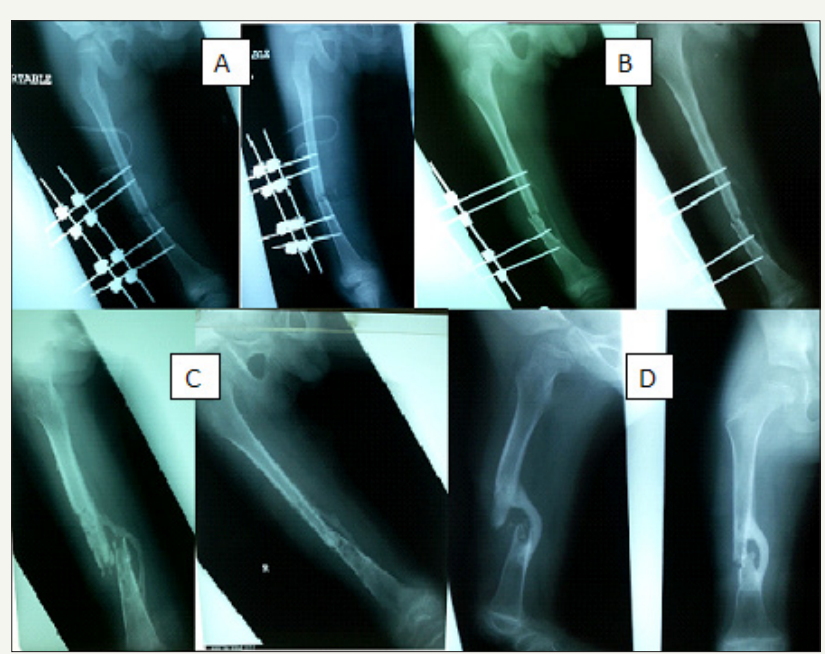

Figure 1: Serial radiographs of the patient's right femur in anteroposterior (AP) and lateral views: 1a: Day 1 post-external fixation; 1b: Osteomyelitic changes of the right femur 2 months post-trauma;

1c: Post-wound debridement, bone resection and external fixator removal;

1d: The fracture site was mobile even though there was medial bridging callus seen on the radiographs at 6 months post-trauma. 
$\mathrm{XY}$, an 8-year-old boy, sustained an open fracture of the right femur grade IIIa in a motor vehicle accident. The patient presented late to the hospital and hence intravenous antibiotics (cefuroxime and metronidazole) were started 4 hours post-trauma. Wound debridement and external fixation (Figure 1a) of the fracture were done more than 12 hours post-trauma. He was given 2 weeks of intravenous antibiotics in ward and was then discharged home well. Wound coverage with split skin graft was done 1-month posttrauma.

Post-trauma 2 months, persistent serous discharge was noted from the pin sites. The child was afebrile. Radiographs of the right femur showed osteomyelitic changes at the pin sites (Figure 1b).
The child was then treated with intravenous cloxacillin and fusidic acid. Wound debridement, external fixator removal and infected bone resection were done. Skeletal traction via a right calcaneal pin was applied. Bone tissue culture revealed a heavy growth of Staphylococcus aureus. The antibiotics were given for a total duration of 6 weeks ( 2 weeks intravenously and 4 weeks via oral route). The right lower limb skeletal traction and calcaneal pin were removed 6 weeks after insertion and a right lower limb full-length thermoplastic splint was applied (Figure 1c). The child was active, ambulating via wheelchair upon discharge with his erythrocyte sedimentation ratio (ESR) improved from 34 to $4 \mathrm{~mm} / \mathrm{hr}$ and white blood cell (WBC) count from 15.23 to $6.1 \times 10^{9} / \mathrm{L}$.

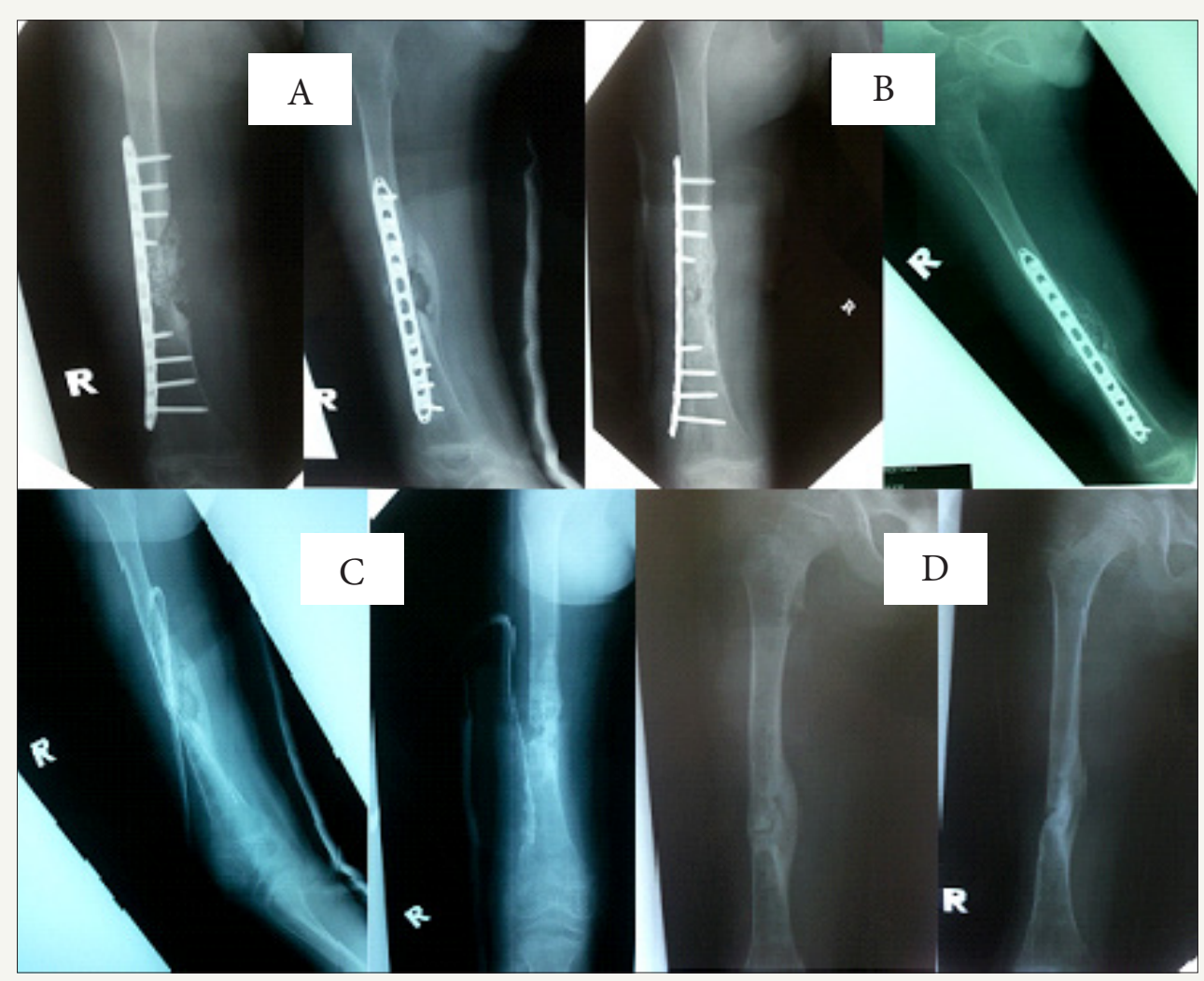

Figure 2: Serial radiographs of the patient's right femur:

2a: Post-3.5mm straight locking plate insertion and bone grafting;

2b: Bridging callus seen 3 months post-LP insertion and bone grafting (9 months post-trauma);

2c: Post-removal of LP;

2d: Remodeling of the right femur at 11 months post-trauma.

During review at six months post-trauma, examination showed a right lower limb shortening of $3 \mathrm{~cm}$ and the fracture site was mobile even though there was medial bridging callus seen on the radiographs (Figure 1d). The wound was clean and septic

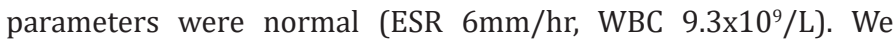
decided to put a temporary 8 -holes $3.5 \mathrm{~mm}$ straight locking plate and $5 \mathrm{~mL}$ synthetic bone graft to hasten the union of the fracture and to reduce the true shortening (Figure 2a). Acute distraction of $2 \mathrm{~cm}$ was done intra-operatively to reduce the true shortening to $1 \mathrm{~cm}$ compared to the contralateral lower limb. The child was given another 2 weeks of oral cloxacillin post-operation with daily wound dressing.
Three months post-plating and bone grafting, bridging callus was seen at the fracture site on the right femoral radiographs (Figure 2b). We decided to remove the implant to prevent another episode of implant infection (Figure 2c). There was right lower limb true shortening of $1 \mathrm{~cm}$ after implant removal. Two months postimplant removal (about eleven months post-trauma), the boy was allowed full weight bearing on his right lower limb with intensive limb physiotherapy (Figure 2d). At 17 months post-trauma, the fracture site was well united with no limb length discrepancy (Figure 3). The range of motion of his right hip and right knee was normal. 




Figure 3: A fully united right femur with no limb-length discrepancy compared with the contralateral femur at 17 months posttrauma.

\section{Discussion}

Open fractures of long bones in children are different from those in adults. They tend to heal faster and are more stable due to thicker and more active periosteums [1]. Infection rate is lower in open fractures of children compared with adults [1]. In this case, there is a high risk of fracture site infection due to grade III open fracture and delayed antibiotic initiation. Open fracture GustilloAnderson grade III has a higher risk of infection (8\%) compared to Gustillo-Anderson grade II (2\%) and I (2\%) [1]. Early initiation of antibiotics (less than three hours after injury) reduces the infection rate from $7.4 \%$ to $4.7 \%$ [1].

In this child, he developed osteomyelitis two months after the initial surgical debridement and external fixation. Bone tissues from the repeated wound debridement cultured a heavy growth of Staphylococcus aureus which is the most common infective organism in this age group [2]. Cloxacillin is the antibiotics of choice for the treatment of osteomyelitis in this group of patients [2]. From the literature, the duration of antibiotics ranges from four to twenty-four weeks, but it is generally accepted that six weeks of antibiotics is adequate after a surgical debridement. In this patient we decided to give antibiotics two weeks intravenously and four weeks via oral route as literatures suggested that oral antibiotics can be as effective as parenteral treatments [2].

Six months post-trauma, there was no clinical sign of union after a trial of conservative management with limb-length discrepancy. In patients with infected non-union of long bones and limb shortening of less than $4 \mathrm{~cm}$, the treatment of choice is single-stage debridement and bone grafting with fracture stabilization $[3,4]$. Fracture can be stabilized with a cast, an external fixator or bone transport (for patients with shortening of more than $4 \mathrm{~cm}$ ) until the healing is completed $[3,4]$. In this patient with a limb shortening of $3 \mathrm{~cm}$, we decided to use a temporary $3.5 \mathrm{~mm}$ straight locking plate (LP) for fracture stabilization. This is more convenient for the patient in terms of wound care and psychologically as compared to external fixation and it allows earlier mobilization in comparison to a cast.
A locking plate is preferred over a conventional plate because:

1. It provides a stable fixation without loosening which might trigger an inflammatory response;

2. It does not compress on the underlying periosteum and will not disrupt the vascularity;

3. It is made of titanium which is associated with a lower risk of infection.

We only partially correct the true shortening $(2 \mathrm{~cm})$ since bones tend to overgrow in children. Literature shows that the earliest removal of LP is 5 months (average 13 months) post- insertion [5]. However, in this boy, we removed the implant early after noted callus formation to prevent another episode of implant infection. We take into consideration the risk of fracture recurrence after the removal of LP, hence a full-length cast was applied for 2 months before allowing full weight bearing [5]. There was limb shortening of $1 \mathrm{~cm}$ post-LP removal, but the bone re-modelled and the child subsequently recovered with no limb-length discrepancy. The range of motion of both right hip and knee was also normal. This case report highlights an alternative technique in treating an infected non-union with limb-length discrepancy, but options such as cast and external fixation may be beneficial in other cases. Hence, the treatment option should be tailored to the patient based on a sound clinical judgement.

\section{Conclusion}

The management of infected non-union after open fractures with limb shortening is challenging. A temporary straight locking plate acting as an internal external fixator and bone graft substitute after surgical debridement can be one of the treatment options.

\section{References}

1. Stewart DGJ, Kay RM, Skaggs DL (2005) Open fractures in children. principles of evaluation and management. JBJS 87(12): 2784-2798.

2. Lazzarini L, Lipsky BA, Mader JT (2005) Antibiotic treatment of osteomyelitis: what have we learned from 30 years of clinical trials? International Journal of Infectious Diseases 9(3): 127-138. 
3. Spiegel DA, Penny JN (2005) Chronic osteomyelitis in children techniques in orthopaedics 20(2): 142-152.

4. Jain AK, Sinha S (2005) Infected nonunion of the long bones. Clin Orthop Relat Res 431: 57-65.
5. Becker T, Weigl D, Mercado E, Katz K, Bar-On E (2012) Fracture and refractures after femoral locking compression plate fixation in children and adolescents. Journal of Pediatric Orthopaedics 32(7): e40-e46.

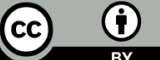

Creative Commons Attribution 4.0 International License

For possible submissions Click Here
Submit Article

Orthopedic Research Online Journal
Benefits of Publishing with us

